ITP-SB-92-70

CEBAF-TH-92-32

\title{
QCD SUM RULES AND COMPTON SCATTERING
}

\author{
Claudio Corianò ${ }^{1,4 *}$, Anatoly Radyushkin ${ }^{2,3 * *}$ and George Sterman ${ }^{1}$ \\ ${ }^{1}$ Institute for Theoretical Physics, State University of New York, \\ Stony Brook, New York 11794-3840 \\ 2 Physics Department, Old Dominion University, Norfolk, VA 23529 \\ ${ }^{3}$ Continuous Electron Beam Accelerator Facility, Newport News, VA 23606 \\ ${ }^{4}$ Department of Physics, University of Stockholm \\ S 113 Vanadisvagen, Stockholm, Sweden
}

\begin{abstract}
We extend QCD sum rule analysis to moderate energy fixed angle Compton scattering. In this kinematic region there is a strong similarity to the sum rule treatment of electromagnetic form factors, although the four-point amplitude requires a modification of the Borel transform. To illustrate our method, we derive the sum rules for helicity amplitudes in pion Compton scattering and estimate their large- $t$ behavior in the local duality approximation.
\end{abstract}

(*) A. Della Riccia fellow

${ }^{(* *)}$ On leave from the Laboratory of Theoretical Physics, JINR, Dubna, Russian Federation 


\section{Introduction}

The method of QCD sum rules [i] originated from an analysis of the high energy behavior of two-point correlation functions of QCD currents. More than 10 years ago, this method was extended to elastic

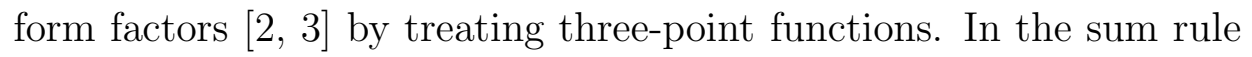
method, one considers Green functions of interpolating fields given by current-correlators which have nonzero resonant contribution on selected lowest states. The non-perturbative resonant region is then

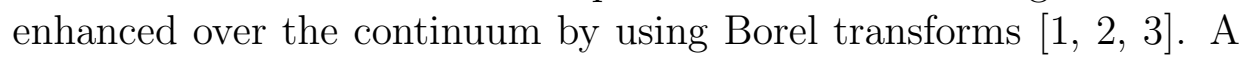
problem in the more general cases [4] involving four or higher-point functions is the larger number of invariants on which the function depends. In this paper, our goal is to extend the QCD sum rule approach to fixed angle pion Compton scattering, a process described by an amplitude which is closely related to the pion electromagnetic form factor.

Our considerations build on previous work on sum rules for the

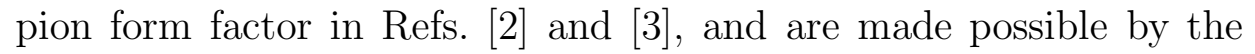
observation that, at high energy and fixed angle, the underlying quarkphoton scattering subprocess has a short-distance nature.

The approach that we investigate is also related to the observation (see, e.g., [1] [ variant of the finite-energy sum rules analogous to those applied orig-

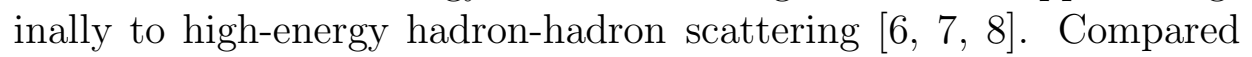
to the sum rule treatment of form factors [i2, 15in, we must take into account the presence of additional cuts in the $u$-channel when we construct a dispersion relation. These additional cuts force us to work in a kinematical regime in $s$ and $t$ where the cuts are far from the region enclosed by the integration contour. Our aim is to show that if we choose $s, t$ and $s+t$ to be moderately large in the physical region $s>0, t<0$, then a dispersion relation can be written down and, hence, a QCD sum rule can be derived. These sum rules result in invariant amplitudes with $s$ and $t$ dependence in general. This dependence is not expected to be the same as for perturbative calculations based on elastic scattering factorization theorems $[\overline{\underline{9}} \mathbf{i}]$, because the sum rule calculation begins at $O\left(\alpha_{s}^{0}\right)$. Thus, when our method is extended to the proton, it may be possible to confront both the 
sum rule and the standard perturbative calculations with experiment at a fixed energy. This would help clarify some controversial issues concerning the relative importance of these contributions at available

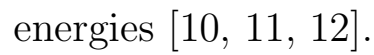

Our arguments are illustrated by an explicit calculation of the discontinuity in the perturbative amplitude at the lowest order, and by a derivation of the sum rules for invariant amplitudes in the case of Compton scattering of the pion.

In Section 2, we reformulate the QCD sum rule approach to the pion electromagnetic form factor. To this end, we modify the standard

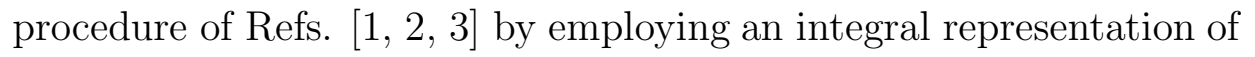
the Borel transform. In Section 3, we develop the relevant dispersion relation for the four-point amplitudes, and derive a sum rule by using a variation of the Borel transform. In Section 4, we discuss the isolation of the pion contribution. Section 5 contains our results for the lowest order spectral functions and the QCD sum rule estimate for the Compton amplitude in the "local duality" approximation. Our conclusions are summarized in Section 6.

\section{Form Factor Sum Rules}

The most popular choice of the interpolating fields in the pion case is the axial current

$$
\eta_{\mu}=\bar{u}(x) \gamma_{\mu} \gamma_{5} d(x)
$$

whose projection onto a single-pion state $|p\rangle$ is given by

$$
\left\langle 0\left|\eta_{\mu}(x)\right| p\right\rangle=i f_{\pi} p_{\mu} e^{-i p \cdot x} .
$$

The electromagnetic current $J_{\mu}$ defining the pion form factor is given by

$$
J_{\mu}=\frac{2}{3} \bar{u} \gamma_{\mu} u-\frac{1}{3} \bar{d} \gamma_{\mu} d
$$

To study the form factor, one analyzes the 3-point correlation function of the electromagnetic and two axial currents [2],

$$
\Gamma_{\mu \nu \sigma}\left(p_{1}, p_{2}\right)=\int\left\langle 0\left|T\left(\eta_{\sigma}(y) J_{\nu}(0) \eta_{\mu}^{\dagger}(x)\right)\right| 0\right\rangle e^{-i p_{1} x+i p_{2} y} d^{4} x d^{4} y
$$


Analogously, to investigate pion Compton scattering, one should consider the 4-point function involving two axial and two electromagnetic currents

$$
\begin{aligned}
\Gamma_{\nu \lambda \mu \sigma}\left(p_{1}, p_{2}, s, t\right)= & i \int d^{4} x d^{4} y d^{4} z e^{-i p_{1} \cdot x+i p_{2} \cdot y-i q_{1} \cdot z} \\
& \times\left\langle 0\left|T\left(\eta_{\sigma}(y) J_{\nu}(z) J_{\lambda}(0) \eta_{\mu}^{\dagger}(x)\right)\right| 0\right\rangle,
\end{aligned}
$$

where the photon lines are assumed to be on-shell.

Let $T_{3}\left(p_{1}^{2}, p_{2}^{2}, t\right)$, with $t=\left(p_{2}-p_{1}\right)^{2}$, denote one of the scalar

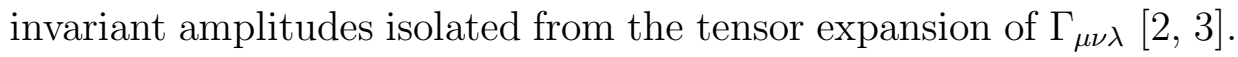
The amplitude $T_{3}$ obeys the double dispersion relation

$$
T_{3}\left(p_{1}^{2}, p_{2}^{2}, t\right)=\int_{0}^{\infty} d s_{1} \int_{0}^{\infty} d s_{2} \frac{\Delta_{3}\left(s_{1}, s_{2}, t\right)}{\left(s_{1}-p_{1}^{2}\right)\left(s_{2}-p_{2}^{2}\right)}+\text { subtractions }
$$

where $\Delta_{3}\left(s_{1}, s_{2}, t\right)$ is the double discontinuity of $T_{3}$ across the branch cut associated with thresholds in $p_{1}^{2}$ and $p_{2}^{2}$.

The next step within the QCD sum rule approach is to introduce the double "Borel" transform $\Phi_{3}\left(M_{1}^{2}, M_{2}^{2}, t\right)\left[\begin{array}{l}2 \\ n\end{array}\right.$, 茂 of $T_{3}\left(p_{1}^{2}, p_{2}^{2}, t\right)$. The Borel transformation can be defined in different ways. For our purposes, the most convenient is the integral representation

$$
\Phi_{3}\left(M_{1}^{2}, M_{2}^{2}, t\right)=\frac{1}{(2 \pi i)^{2}} \int_{C} \frac{d p_{1}^{2}}{M_{1}^{2}} \int_{C} \frac{d p_{2}^{2}}{M_{2}^{2}} e^{-p_{1}^{2} / M_{1}^{2}-p_{2}^{2} / M_{2}^{2}} T_{3}\left(p_{1}^{2}, p_{2}^{2}, t\right),
$$

where $C$ denotes any contour that encloses the branch cuts in the $p_{1}^{2}$ and $p_{2}^{2}$ planes, from zero to infinity. For any non-zero values of the $M_{i}^{2}$, the contours $C$ may be thought of as closed across the cut at positive infinity, where the integrand is exponentially suppressed.

For the 3-point function, the integral form (iii) is completely equiv-

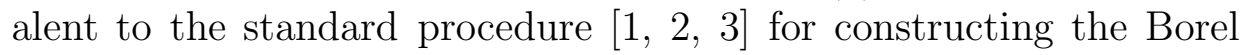
transform. However, as we will see, it is very suggestive of an extension to the four-point function related to fixed-angle high-energy pion Compton scattering.

Note that eq. (iii) immediately gives zero result for the subtraction terms in $T_{3}\left(p_{1}^{2}, p_{2}^{2}, t\right)$, since they are polynomials in the $p_{i}^{2}$. Applying 


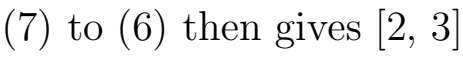

$$
\Phi_{3}\left(M_{1}^{2}, M_{2}^{2}, t\right)=\int_{0}^{\infty} \frac{d s_{1}}{M_{1}^{2}} \int_{0}^{\infty} \frac{d s_{2}}{M_{2}^{2}} \Delta_{3}\left(s_{1}, s_{2}, t\right) e^{-s_{1} / M_{1}^{2}-s_{2} / M_{2}^{2}} .
$$

To derive a sum rule, we employ our freedom in choosing the original contours $C$ in the definition of the double transform, eq. (iii). Two relevant choices are shown in Fig. 1. Contour $C_{a}$ encloses the branch cut closely, while $C_{b}$ encloses it with a circle of radius $\zeta^{2}$ about the origin, and joins with $C_{a}$ for $\left|p^{2}\right|>\zeta^{2}$. Let us suppose that we choose

$$
\zeta^{2} \gg \mu^{2}
$$

where $\mu$ is the scale associated with nonperturbative power corrections in $T_{3}\left(p_{1}^{2}, p_{2}^{2}, t\right)$. The inequality makes it reasonable to apply the operator product expansion along $C_{b}$. Around the contour $C_{b}$, we may now approximate

$$
T_{3}\left(p_{1}^{2}, p_{2}^{2}, t\right)=T_{3}^{\text {pert }}\left(p_{1}^{2}, p_{2}^{2}, t\right)+\text { power corrections, }
$$

where $T_{3}{ }^{\text {pert }}\left(p_{1}^{2}, p_{2}^{2}, t\right)$ is the perturbative expansion for $T_{3}$ starting with the diagrams of Fig. 2. The power corrections indicated in eq. (10 are proportional to the familiar vacuum condensates,

$$
\text { power corrections }=K_{1} \frac{\left\langle 0\left|\alpha_{s} G_{\mu \nu} G^{\mu \nu}\right| 0\right\rangle}{\left(p_{1}^{2}\right)^{2}}+K_{2} \frac{\left\langle 0\left|\alpha_{s}(\bar{q} q)^{2}\right| 0\right\rangle}{\left(p_{1}^{2}\right)^{3}}+\ldots \text {, }
$$

with $K_{1}$ and $K_{2}$ being, in general, functions of $t / p_{1}^{2}$ and $p_{1}^{2} / p_{2}^{2}$. We shall assume that $\zeta^{2}=O(t)$ in eq. $(\overline{\underline{n}})$, so that possible higher-order logarithmic corrections in these variables are not large.

Around the contour $C_{a}$, the approximation of eq. (1) $(\bar{Q})$ is clearly inappropriate. Instead, for small values of $p_{1}^{2}$ and $p_{2}^{2}$, we parameterize $T_{3}$ phenomenologically - in terms of dominant resonances plus continuum:

$$
\Delta_{3}\left(p_{1}^{2}, p_{2}^{2}, t\right)=\Delta_{3}^{p h e n}\left(p_{1}^{2}, p_{2}^{2}, t\right) .
$$

The sum rules now result from eq. (iי (i) by equating the two transforms, one around $C_{a}$ and one around $C_{b}$, using the two approximations, eqs.

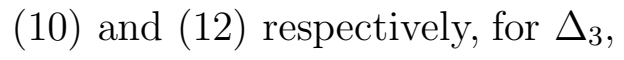

$$
\begin{gathered}
0=\int_{0}^{\infty} \frac{d s_{1}}{M_{1}^{2}} \int_{0}^{\infty} \frac{d s_{2}}{M_{2}^{2}}\left(\Delta_{3}^{\text {pert }}-\Delta_{3}^{\text {phen }}\right) e^{-s_{1} / M_{1}^{2}-s_{2} / M_{2}^{2}} \\
+ \text { power corrections, }
\end{gathered}
$$


with $\Delta_{3}{ }^{\text {pert }}$ the double discontinuity of $T_{3}{ }^{\text {pert }}$ around the $p_{1}^{2}$ and the $p_{2}^{2}$ cuts. Here the contributions of power corrections will appear only from the (counterclockwise) circle of radius $\zeta^{2}$ for $C_{a}$ in Fig. 1,

$$
\frac{1}{2 \pi i} \int_{\left|p^{2}\right|=\zeta^{2}} \frac{d p^{2}}{M^{2}} \frac{e^{-p^{2} / M^{2}}}{\left(p^{2}\right)^{n}}=\frac{(-1)^{n}}{(n-1) !\left(M^{2}\right)^{n}} .
$$

The remainder of the $C_{a}$ integral gives zero for power corrections, since they are well approximated by functions that are analytic except at the origin. Of course, this is an approximation, but an adequate one

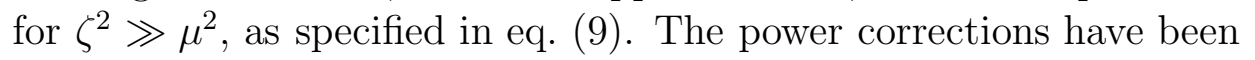
computed in Refs. [2] eq. (1, $(\overline{3})$, information can be extracted when an explicit form is given for $\Delta_{3}$ phen. For the form factor, the standard choice "first resonance

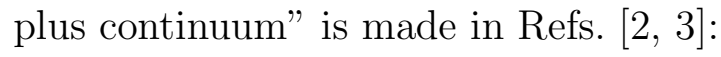

$$
\begin{aligned}
\Delta^{\text {phen }}\left(s_{1}, s_{2}, t\right)= & T_{3}^{\pi}(t) \delta\left(s_{1}-m_{\pi}^{2}\right) \delta\left(s_{2}-m_{\pi}^{2}\right) \\
& +\Delta_{3}{ }^{\text {pert }}\left(s_{1}, s_{2}, t\right)\left(1-\theta\left(s_{0}-s_{1}\right) \theta\left(s_{0}-s_{2}\right)\right),
\end{aligned}
$$

where $T_{3}^{\pi}(t)$ is the pionic scalar amplitude and $s_{0}$ is a parameter spec-

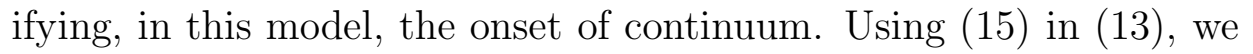
find the $M^{2}$ - and $s_{0^{-}}$dependent sum rule,

$$
\begin{gathered}
T_{3}^{\pi}(t)=\int_{0}^{s_{0}} d s_{1} \int_{0}^{s_{0}} d s_{2} \Delta_{3}^{\text {pert }}\left(s_{1}, s_{2}, t\right) e^{-\left(s_{1}+s_{2}\right) / M^{2}} \\
+ \text { power corrections, }
\end{gathered}
$$

where we have set $M_{1}^{2}=M_{2}^{2}=M^{2}$ and neglected $m_{\pi}^{2}$ compared to all other scales.

Note that contributions from the states beyond $\sqrt{s_{0}}$ in mass have cancelled here. Fixing $s_{0}$ from the requirement of the best stability of the right hand side of eq. (1 $1 \overline{6} \bar{i})$ with respect to variations of the Borel parameter $M^{2}$ (this gives $s_{0} \approx 0.7 \mathrm{GeV}^{2}$ ) and then taking the limit $M^{2} \rightarrow \infty$ (which eliminates power corrections), one arrives at the "local duality" formula for the pion form factor [3i]. We now turn to the application of this reasoning to the four-point amplitude. 


\section{Sum Rules for Four-Point Amplitudes}

In general, the analytic structure of a four particle scattering amplitude, or a four-current correlation function, is much more complicated than that of a three-point function. Nevertheless, at high energy and momentum transfer, the space-time structure of the Compton scattering amplitudes simplifies somewhat, and becomes similar to the corresponding form factor amplitudes.

Consider, for instance, the four-current Green function $\Gamma_{\nu \lambda \mu \sigma}$, eq.

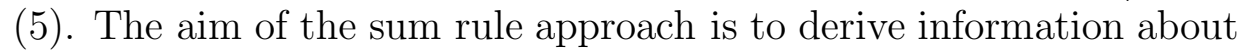
$\Gamma_{\nu \lambda \mu \sigma}$ at $p_{i}^{2}=m_{\pi}^{2}$, where this function develops bound-state poles. Of course, we cannot compute $\Gamma$ perturbatively at these momenta. If, however, we can use a dispersion representation to relate the Green function in this nonperturbative region to its behavior in a region where perturbation theory and the operator product expansion may be applied, the road is open to apply the methods described in the previous section. We shall argue that it is possible to do this quite directly when the Mandelstam invariants $s=\left(p_{1}+q_{1}\right)^{2}$ and $t=$ $\left(p_{1}-p_{2}\right)^{2}$ in eq. (․․․

The first step in this process is to note that even for on-shell external particles, the underlying hard scattering at fixed $s / t$ remains a short-distance process in any two-to-two scattering amplitude [i[3] Perturbation theory runs into trouble from virtual particles that either (1) are on-shell and parallel to one of the four external momenta (collinear lines), or (2) have vanishing momenta in all four components (soft lines). Momentum configurations of this type will typically diverge in the zero-mass limit, or, equivalently, suffer from large logarithms of ratios of external energies to masses. The situation for Compton scattering at lowest order in electromagnetism, however, simplifies when the external photons carry physical polarizations. In this case, virtual lines parallel to the incoming or outgoing photons do not produce large logarithms, nor do soft lines attached to such collinear lines. The failure of perturbation theory, then, results from the lines that are collinear to one of the hadron momenta and/or from the soft quanta that connect them. This is qualitatively the same situation as for the elastic form factor. Indeed, in space-time, the incoming photon and the outgoing photon originate at the same 
point. This observation has been used elsewhere in the context of a perturbative QCD calculation of Compton scattering to show that the phases of this amplitude are free of infrared divergences [1] $\left.{ }_{1}^{1} \mathbf{1}_{1}\right]$.

To take advantage of these observations, we shall need to develop the relevant dispersion relation. Its form will be slightly different from the form factor case, requiring the modified version of the Borel transform mentioned above. Let us describe how this works.

Let $T_{4}\left(p_{1}^{2}, p_{2}^{2}, s, t\right)$ denote a particular scalar amplitude derived from $\Gamma_{\nu \lambda \mu \sigma}$ in eq. ( $\left({ }_{1}^{\prime}\right)$. We will give a specific example below. Momentum assignments are illustrated in Fig. 3. We will be interested in $s$ and $t$ large compared to $m_{\pi}^{2}$, with $-t / s$ fixed but not equal to 1 or 0 . We take $q_{1}^{2}=q_{2}^{2}=0$, appropriate to physical photons.

In general, we are not able to write for $T_{4}$ a double dispersion integral of precisely the form of eq. ( $p_{2}^{2}$ becomes large enough, the kinematic identity

$$
s+t+u=p_{1}^{2}+p_{2}^{2}
$$

forces $u$ to threshold for fixed $s$ and $t$. This means, first, that branch points in $u$ appear at $p_{2}^{2}$-dependent positions in the $p_{1}^{2}$ plane (and vice-versa). Second, it means that, even at large $p_{1}^{2}+p_{2}^{2}$, a phenomeno-

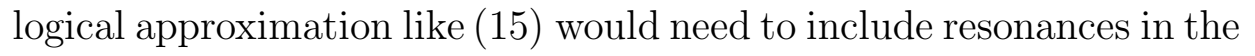
$u$-channel. Fortunately, this is not the end of the story. It is still possible to write a useful double dispersion relation for $T_{4}$. This relation employs the contour $\gamma$ shown in Fig. 4.

Instead of running to infinity as in eq. (i, $\left.\mathbf{6}_{-1}^{i}\right), \gamma$ is closed in a circle of radius $\lambda^{2}$, where $\lambda^{2}$ is a constant which we may take to satisfy

$$
\lambda^{2}=(s+t) / 4 \text {. }
$$

With this choice, the $u$-channel threshold never comes closer than $(s+t) / 2$ to $\gamma$ in the $p_{1}^{2}$ plane, when $p_{2}^{2}$ is chosen to run along the corresponding contour in its complex plane. We therefore have, in

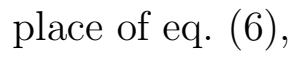

$$
T_{4}\left(p_{1}^{2}, p_{2}^{2}, s, t\right)=\frac{1}{(2 \pi i)^{2}} \int_{\gamma} d s_{1} \int_{\gamma} d s_{2} \frac{T_{4}\left(s_{1}, s_{2}, s, t\right)}{\left(s_{1}-p_{1}^{2}\right)\left(s_{2}-p_{2}^{2}\right)} .
$$

We note that the portion of $\gamma$ that runs along the real axis gives an integral over a discontinuity, as in $\left(\overline{\mathbf{b}_{1}}\right)$, but, generally speaking, the 
discontinuity is not purely imaginary. Hence, the amplitude we are interested in will be complex in general, even for moderate negative $p_{1}^{2}$ and $p_{2}^{2}$. It remains, however, an analytic function of $s$ if we are far from its thresholds.

More formal arguments for the validity of this dispersion relation, and for the applicability of perturbation theory to the amplitude at large values of $\left|p_{i}^{2}\right|$ are given in Appendix A. Here we shall assume that these results hold, and show how they lead to the desired sum rule.

To derive a sum rule, we would like to apply the same Borel trans-

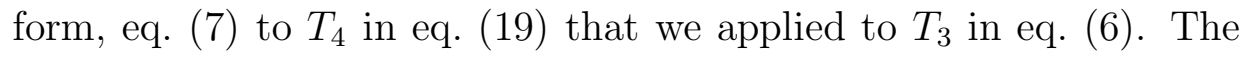
contours $C$ in eq. ( $(\bar{i})$, however, enclose the entire real axis, and would

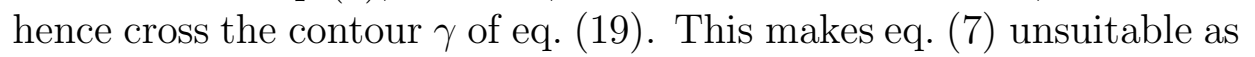
it stands. This technical difficulty can be circumvented if we choose a slightly modified version of the transform. As shown in Fig. 4, let $\Gamma$ be any counterclockwise contour, enclosing the positive real axis of the $p_{i}^{2}$ plane, which starts at $\lambda^{2}-i \epsilon$ and ends at $\lambda^{2}+i \epsilon$, but remains inside the overall contour $\gamma$ of Fig. 4. Note that $\Gamma$ is not quite closed on the real axis. We assume that $s$ and $t$ are large enough so that we may choose

$$
\lambda^{2} \gg M_{i}^{2} \gg \mu^{2}
$$

with $\mu$ the scale of power corrections, $\lambda^{2}$ given by eq. (i $\overline{1} \overline{8}$ i) and $M_{i}$ the arguments of the transform. We then define our modified "Borel" transform of $T_{4}$ by

$$
\begin{aligned}
\Phi_{4}\left(M_{1}^{2}, M_{2}^{2}, s, t\right) \equiv & \frac{1}{(2 \pi i)^{2}} \int_{\Gamma} \frac{d p_{1}^{2}}{M_{1}^{2}} \int_{\Gamma} \frac{d p_{2}^{2}}{M_{2}^{2}} e^{-p_{1}^{2} / M_{1}^{2}}\left(1-e^{-\left(\lambda^{2}-p_{1}^{2}\right) / M_{1}^{2}}\right) \\
& \times e^{-p_{2}^{2} / M_{2}^{2}}\left(1-e^{-\left(\lambda^{2}-p_{2}^{2}\right) / M_{2}^{2}}\right) T_{4}\left(p_{1}^{2}, p_{2}^{2}, s, t\right) \\
= & \frac{1}{(2 \pi)^{2}} \int_{0}^{\lambda^{2}} \frac{d s_{1}}{M_{1}^{2}} \int_{0}^{\lambda^{2}} \frac{d s_{2}}{M_{2}^{2}} \Delta_{4}\left(s_{1}, s_{2}, s, t\right) e^{-s_{1} / M_{1}^{2}-s_{2} / M_{2}^{2}} \\
& \times\left(1-e^{-\left(\lambda^{2}-s_{1}\right) / M_{1}^{2}}\right)\left(1-e^{-\left(\lambda^{2}-s_{2}\right) / M_{2}^{2}}\right)
\end{aligned}
$$

In the second equality, the evaluation of the $p_{i}^{2}$ integrals has been carried out, using Cauchy's theorem and the representation eq. (1, $\left.\overline{1}_{1}^{\bar{g}}\right)$ for $T_{4}$, by simply shrinking the $\Gamma$ contours to the fixed points $\lambda^{2} \pm i \epsilon$. For $s_{i}$ real and $\leq \lambda^{2}$, we pick up a pole contribution, just as in eq. (1 
for the three-point function, while for $s_{i}$ on the circular part of the $\gamma$ contour in Fig. 4, we get no contribution, as the $\Gamma$ contour shrinks to the infinitesimal segment between $\pm i \epsilon$ without encountering any singularities. At the special points, $s_{i}=\lambda^{2}$, the factor $1-e^{-\left(\lambda^{2}-p_{i}^{2}\right) / M_{i}^{2}}$ vanishes at the $p_{i}^{2}=\lambda^{2}$ pole, so that the transform gets a vanishing contribution from these points as well. As usual, $\Delta_{4}$ is the double discontinuity of $T_{4}$. From eq. ( $\left.2 \overline{1} \overline{1} \overline{1}\right)$, we will derive sum rules exactly analogous to the form factor result, eq. ('1 $1 \overline{1} \overline{3})$, by equating the perturbative plus power corrections expression for $T_{4}\left(s_{1}, s_{2}, s, t\right)$ with a phenomenological parameterization of $\Delta_{4}$ in eq. (19). Let us see how this works for the case at hand, the Compton scattering of a pion.

\section{The Pion Compton Amplitude}

The pion matrix element

$$
M_{\nu \lambda}=i \int d^{4} y e^{-i q_{1} y}\left\langle p_{2}\left|T\left(J_{\nu}(y) J_{\lambda}(0)\right)\right| p_{1}\right\rangle
$$

is the object we will study in terms of its invariant amplitudes, for which we may construct sum rules as in the previous section. $M_{\nu \lambda}$ can be expressed in terms of two invariant amplitudes. We use helicity form factors $H_{1}$ and $H_{2}$ defined by the relation [i]

$$
M^{\lambda \mu}=H_{1} e^{(1) \lambda} e^{(1) \mu}+H_{2} e^{(2) \lambda} e^{(2) \mu},
$$

with

$$
\begin{gathered}
e^{(1) \lambda}=\frac{N^{\lambda}}{\sqrt{-N^{2}}} \quad e^{(2) \lambda}=\frac{P^{\lambda}}{\sqrt{-P^{2}}}, \\
N^{\lambda}=\epsilon^{\lambda \mu \nu \rho} P_{\mu} r_{\nu} R_{\rho} \quad P^{\lambda}=p_{1}^{\lambda}+\nu p_{2}^{\lambda}-R^{\lambda} \frac{p_{1} \cdot R+\nu p_{2} \cdot R}{R^{2}},
\end{gathered}
$$

where

$$
\nu=\frac{p_{1} \cdot p_{2}-s_{1}}{p_{1} \cdot p_{2}-s_{2}}
$$

and where

$$
R=q_{1}+q_{2} \quad r=q_{2}-q_{1} .
$$


The "polarization" vectors $e^{(i)}$ satisfy

$$
\begin{aligned}
e^{(i)} \cdot q_{1} & =e^{(i)} \cdot q_{2}=0, \\
e^{(i)} \cdot e^{(j)} & =-\delta_{i j} .
\end{aligned}
$$

Note that these relations hold for all positive $s_{1}=p_{1}^{2}$ and $s_{2}=p_{2}^{2}$, whether or not they are equal. We also note that $e^{(1)}$ serves as the normal to the scattering plane defined by the Compton process, while $e^{(2)}$ is a unit spacelike vector whose spatial projection is in the scattering plane, but which is orthogonal to the $q_{i}$ 's. Other expansions are also possible, but we choose this one for definiteness and for its simplicity.

Now $M_{\nu \lambda}$ appears as the residue at the double pion pole at $p_{1}^{2}=$ $p_{2}^{2}=m_{\pi}^{2}$ in the four-point Green function $\Gamma_{\nu \lambda \mu \sigma}$ introduced in eq. ( (1) To be more specific, let us define a double discontinuity $\Delta_{\nu \lambda \mu \sigma}$ of $\Gamma_{\nu \lambda \mu \sigma}$ across the $p_{1}^{2}$ and $p_{2}^{2}$ cuts by

$$
\Delta_{\nu \lambda \mu \sigma} \equiv \Gamma_{++}-\Gamma_{+-}-\Gamma_{-+}+\Gamma_{--}
$$

where we define $\Gamma_{++}=\Gamma_{\nu \lambda \mu \sigma}\left(p_{1}^{2}+i \epsilon, p_{2}^{2}+i \epsilon\right)$, and so on. The poles may be isolated from the $x_{0} \rightarrow-\infty, y_{0} \rightarrow \infty$ limit of $\Gamma_{\nu \lambda \mu \sigma}$ in eq. ( ( $\left.\begin{array}{c}5 \\ 1 \\ 1\end{array}\right)$, by inserting complete sets of states in the resulting time ordering, as

$$
\begin{aligned}
\Gamma_{\nu \lambda \mu \sigma}= & \frac{i}{(2 \pi)^{6}} \int d^{4} x d^{4} y d^{4} z d^{4} p^{\prime}{ }_{1} d^{4} p^{\prime}{ }_{2} \theta\left(y_{0}\right) \theta\left(-x_{0}\right) \\
& \times e^{-i p_{1} \cdot x-i q_{1} \cdot z+i p_{2} \cdot y} \\
& \times \delta\left(p^{\prime 2}-m_{\pi}^{2}\right) \delta\left(p^{\prime}{ }_{2}^{2}-m_{\pi}^{2}\right)\left\langle 0\left|\eta_{\sigma}(y)\right| p_{2}^{\prime}\right\rangle \\
& \times\left\langle p_{2}^{\prime}\left|T\left(J_{\nu}(z) J_{\lambda}(0)\right)\right| p_{1}^{\prime}\right\rangle\left\langle p_{1}^{\prime}\left|\eta_{\mu}^{\dagger}(x)\right| 0\right\rangle+\Gamma^{c o n t}
\end{aligned}
$$

where $\Gamma^{\text {cont }}$ includes contributions from the higher states and other time orderings, which do not contribute to the double pion pole. As usual, we express the matrix elements of the axial currents as

$$
\begin{aligned}
\left\langle 0\left|\eta_{\sigma}(y)\right| p_{2}^{\prime}\right\rangle & =i f_{\pi} p_{2 \sigma}^{\prime} e^{-i p^{\prime}{ }_{2} \cdot y} \\
\left\langle p_{1}^{\prime}\left|\eta_{\mu}^{\dagger}(x)\right| 0\right\rangle & =-i f_{\pi} p_{{ }_{1 \mu}}^{\prime} e^{i p^{\prime}{ }_{1} \cdot x}
\end{aligned}
$$


This relates the single pion states $p_{1}^{\prime}, p_{2}^{\prime}$ to the vacuum at times $\pm \infty$, with $f_{\pi}$ the pion decay constant. We then derive the relation

$$
\begin{gathered}
\Delta_{\nu \lambda \mu \sigma}=f_{\pi}^{2} p_{1 \mu} p_{2 \sigma}(2 \pi)^{2} \delta\left(p_{1}^{2}-m_{\pi}^{2}\right) \delta\left(p_{2}^{2}-m_{\pi}^{2}\right) \\
\times M_{\nu \lambda}\left(p_{1}, p_{2}, q_{1}\right)+\Delta^{c o n t}
\end{gathered}
$$

with $M_{\nu \lambda}$ given by eq. (2) $(\overline{2} \overline{2})$, and with $\Delta^{\text {cont }}$ the double discontinuity of $\Gamma^{\text {cont }}$.

We now relate the tensor $\Delta_{\nu \lambda \mu \sigma}$ to the invariant amplitudes $H_{1}$ and $H_{2}$ of eq. (2) $(2 \overline{3})$. To do so, we begin by defining two projections,

$$
\begin{aligned}
\Gamma^{(12)}\left(p_{1}, p_{2}, q_{1}\right)_{\mu \sigma} & \equiv-g^{\nu \lambda} \Gamma_{\nu \lambda \mu \sigma}, \\
\Gamma^{(1)}\left(p_{1}, p_{2}, q_{1}\right)_{\mu \sigma} & \equiv e^{(1)^{\nu}} e^{(1)^{\lambda}} \Gamma_{\nu \lambda \mu \sigma} .
\end{aligned}
$$

When the pion poles in $\Gamma^{(12)}$ and $\Gamma^{(1)}$ are isolated, their coefficients will be proportional to $H_{1}+H_{2}$ and $H_{1}$, respectively. Thus, we want to identify a set of tensor structures in $\Gamma^{(12)}$ and $\Gamma^{(1)}$ in which the pion poles may appear.

Note that $\Delta_{\nu \lambda \mu \sigma}$ may be expanded in terms of tensors made from $g^{\alpha \beta}$ and its external vectors, $q_{i}$ and $p_{i}$. Following Refs. [i-i] and [iji] we choose to expand in a basis made from the independent vectors $p_{1}+p_{2}, p_{1}-p_{2}$ and $q_{1}$. (The tensors $e_{\mu}^{(i)} e_{\sigma}^{(i)}$ built from the vectors in eq. (24) may be expanded in this basis as well, when we include the metric tensor.) We then seek a vector $n$, which has the properties

$$
\left(n \cdot q_{1}\right)=\left(n \cdot p_{1}\right)-\left(n \cdot p_{2}\right)=n^{2}=0 .
$$

It is easy to verify that no such $n^{\mu}$ exists with real coefficients. In fact we can immediately give an explicit expression for $n^{\mu}$ in terms of the polarization vectors $e^{(i)}$ above, as

$$
n^{\mu}=\left(e^{(2)} \pm i e^{(1)}\right)^{\mu} .
$$

The specific values of the $n^{\mu}$ are frame-dependent of course, and we need not give them here. We note, however, that either choice of sign in eq. (3.7il) will give the same (real) answers below. The overall normalization of $n^{\mu}$ also cancels in the sum rule result. 
If we saturate the $\mu$ and $\sigma$ indices with an $n$ that satisfies eq. ( $\left.\overline{3} \overline{6}_{1}^{i}\right)$, we will project onto the tensor structure $\left(p_{1}+p_{2}\right)_{\mu}\left(p_{1}+p_{2}\right)_{\sigma}$ only, whose corresponding invariant functions we denote as

$$
\begin{aligned}
\Gamma_{4}^{(12)}\left(p_{i}^{2}, s, t\right) & \equiv n^{\mu} n^{\sigma} \Gamma^{(12)}\left(p_{1}, p_{2}, q_{1}\right)_{\mu \sigma} \\
\Gamma_{4}^{(1)}\left(p_{i}^{2}, s, t\right) & \equiv n^{\mu} n^{\sigma} \Gamma^{(1)}\left(p_{1}, p_{2}, q_{1}\right)_{\mu \sigma}
\end{aligned}
$$

Finally, taking as above the double discontinuities, denoted as $\Delta_{4}^{(i)}$, we isolate the desired invariant functions

$$
\begin{aligned}
\Delta_{4}^{(12)}\left(p_{i}^{2}, s, t\right)= & f_{\pi}^{2}\left(n \cdot p_{1}\right)\left(n \cdot p_{2}\right)(2 \pi)^{2} \delta\left(p_{1}^{2}-m_{\pi}^{2}\right) \delta\left(p_{2}^{2}-m_{\pi}^{2}\right) \\
& \times\left(-g^{\nu \lambda} M_{\nu \lambda}\right)+\Delta_{4}^{(12) c o n t} \\
= & f_{\pi}^{2}\left(n \cdot p_{1}\right)\left(n \cdot p_{2}\right)(2 \pi)^{2} \delta\left(p_{1}^{2}-m_{\pi}^{2}\right) \delta\left(p_{2}^{2}-m_{\pi}^{2}\right) \\
& \times\left(H_{1}(s, t)+H_{2}(s, t)\right)+\Delta_{4}^{(12) c o n t}
\end{aligned}
$$

and similarly

$$
\begin{aligned}
\Delta_{4}^{(1)}\left(p_{i}^{2}, s, t\right)= & f_{\pi}^{2}\left(n \cdot p_{1}\right)\left(n \cdot p_{2}\right)(2 \pi)^{2} \delta\left(p_{1}^{2}-m_{\pi}^{2}\right) \delta\left(p_{2}^{2}-m_{\pi}^{2}\right) \\
& \times H_{1}(s, t)+\Delta_{4}^{(1) c o n t} .
\end{aligned}
$$

The sum rules for the $\Gamma_{4}^{(i)}$ are derived as for $\Gamma_{3}$ above. Consider, for instance, $\Gamma_{4}^{(12)}$. First, we approximate $\Gamma_{4}^{(12)}$ by perturbation theory plus power corrections for a choice of contour $\gamma$ in eq. $\left(\overline{1}_{1} \overline{q_{1}}\right)$ that stays away from the real axis, except at $p_{i}^{2}=\lambda^{2}$,

$$
\Gamma_{4}^{(12)}\left(p_{1}^{2}, p_{2}^{2}, s, t\right)=\Gamma_{4}^{(12) p e r t}\left(p_{1}^{2}, p_{2}^{2}, s, t\right)+\text { power corrections. }
$$

This is analogous to eq. (1, $\left(\overline{1} \overline{0}_{1}\right)$ for the three point function. Next, choosing $\gamma$ in eq. ( $(\overline{1} \bar{g})$ on the real axis, we approximate its discontinuity by a "first resonance plus continuum" form, analogous to eq. (1) (1)

$$
\begin{aligned}
\Delta_{4}^{(12)}= & f_{\pi}^{2}\left(n \cdot p_{1}\right)\left(n \cdot p_{2}\right)(2 \pi)^{2} \delta\left(p_{1}^{2}-m_{\pi}^{2}\right) \delta\left(p_{2}^{2}-m_{\pi}^{2}\right) \\
& \times\left(H_{1}(s, t)+H_{2}(s, t)\right) \\
& +\Delta_{4}^{(12) p e r t}\left(1-\theta\left(s_{0}-s_{1}\right) \theta\left(s_{0}-s_{2}\right)\right) .
\end{aligned}
$$


Combining these expressions in the transform of eq. (12 127$)$, we derive the full sum rule as

$$
\begin{aligned}
f_{\pi}^{2} & \left(n \cdot p_{1}\right)\left(n \cdot p_{2}\right)\left(H_{1}(s, t)+H_{2}(s, t)\right) \\
= & \int_{0}^{s_{o}} d s_{1} \int_{0}^{s_{o}} d s_{2} \Delta_{4}^{(12) p e r t}\left(s_{1}, s_{2}, s, t\right) e^{-s_{1} / M_{1}^{2}-s_{2} / M_{2}^{2}} \\
& \quad \times\left(1-e^{-\left(\lambda^{2}-s_{1}\right) / M_{1}^{2}}\right)\left(1-e^{-\left(\lambda^{2}-s_{2}\right) / M_{2}^{2}}\right) \\
& +f_{1}\left(s, t, M_{i}^{2}\right) \frac{\left\langle 0\left|\alpha_{s} G_{\mu \nu} G^{\mu \nu}\right| 0\right\rangle}{\left(M_{1}^{2}\right)^{2}}+f_{2}\left(s, t, M_{i}^{2}\right) \frac{\left\langle 0\left|\alpha_{s}(\bar{q} q)^{2}\right| 0\right\rangle}{\left(M_{1}^{2}\right)^{3}}+\ldots,
\end{aligned}
$$

where we have written out the power corrections schematically, as in eq. $\left(\underline{1} \underline{1}_{1}^{\prime}\right)$. The functions $f_{i}\left(s, t, M_{i}^{2}\right)$ may be calculated as for the three-point function [믈, vestigation.

As in the form factor case of Section 2 above, $s_{0}$ should be fixed by demanding best stability with respect to variations in $M^{2}=M_{1}^{2}=$ $M_{2}^{2}$. We shall assume that the power corrections are small enough so that, as we increase the $M_{i}^{2}$, still keeping $s / M_{i}^{2}$ large, there is a range where they are negligible compared to the perturbative contributions on the right-hand side of eq. ( $(1 \overline{4} \overline{4})$. Then we may write a "local duality" sum rule, without power corrections:

$f_{\pi}^{2}\left(n \cdot p_{1}\right)\left(n \cdot p_{2}\right)\left(H_{1}(s, t)+H_{2}(s, t)\right)=\int_{0}^{s_{o}} d s_{1} \int_{0}^{s_{o}} d s_{2} \Delta_{4}^{(12) p e r t}\left(s_{1}, s_{2}, s, t\right)$.

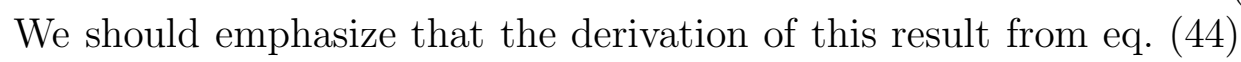
involves assumptions on the behavior of the condensates. The right hand side of eq. ( $\left(\overline{1}_{1}^{4} \overline{\mathbf{S}}_{1}^{\prime}\right)$ is approximated only by the local duality integral, and is to be calculated using perturbation theory. This is the subject of the following section.

\section{Local Duality Sum Rules and Asymp- totic Behavior}

The lowest order perturbative spectral weights $\Delta_{4}^{(i) \text { pert }}$ can be computed from Figs. 5(a) and 5(b) by use of Cutkosky rules. The remaining lowest order diagrams, in which $q_{2}$ and $p_{2}$ are exchanged, do not 
contribute to the double discontinuity in this kinematic region. The double discontinuity in $p_{1}^{2}$ and $p_{2}^{2}$ is found by simply replacing the propagators that carry momenta $k^{\mu},\left(p_{1}-k\right)^{\mu}$ and $\left(p_{2}-k\right)^{\mu}$ by factors $(2 \pi) \delta_{+}\left(k^{2}\right)$, and so on, where the plus indicates that energy flows in the same direction as for $p_{1}$ and $p_{2}$. The procedure is similar to the one adopted in the case of the triangle diagram [2], 'i $1 \overline{6}]$ for 3 -point functions.

We then write $\Delta_{4}^{(i) p e r t}$ as the sum of the two spectral functions $\Delta_{a}^{(i) p e r t}$ and $\Delta_{b}^{(i) p e r t}$ found from the diagrams of Fig. 5, with an overall constant separated out,

$$
\begin{aligned}
\Delta_{4}^{(i) p e r t} & \left(s_{1}, s_{2}, s, t\right) \\
= & \frac{5}{3(2 \pi)^{3}}\left(\Delta_{a}^{(i) p e r t}\left(s_{1}, s_{2}, s, t\right)+\Delta_{b}^{(i) p e r t}\left(s_{1}, s_{2}, s, t\right)\right) .
\end{aligned}
$$

The extra factors are from the flavor structure of the current and the color traces, as well as from the factors of $(2 \pi)^{-1}$ associated with the loop integral. A factor of $(2 \pi)^{-2}$ associated with the dispersion

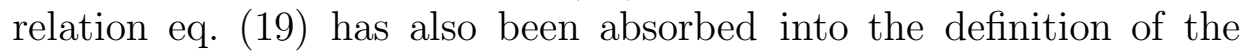
spectral function.

For specific calculation, we found it useful to work in a "brick-wall" frame for $p_{1}$ and $p_{2}$, with $p_{1 \perp}=p_{2 \perp}=0$; the corresponding kinematics is described in some detail in Appendix B. Let us consider the $-g^{\nu \lambda}$ projection, $\Delta_{4}^{(12)}$, related by the sum rule to the combination $H_{1}+H_{2}$.

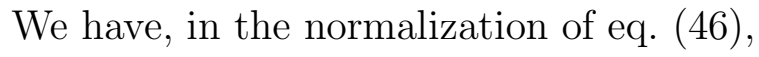

$$
\begin{aligned}
\Delta_{a}^{(12) \text { pert }}=16 & \int d^{4} k \frac{\delta_{+}\left(k^{2}\right) \delta_{+}\left(\left(p_{1}-k\right)^{2}\right) \delta_{+}\left(\left(p_{2}-k\right)^{2}\right)}{\left(p_{1}-k+q_{1}\right)^{2}} \\
\times & (n \cdot k)\left[\left(q_{1} \cdot p_{1}+q_{1} \cdot p_{2}\right)\left(n \cdot p_{1}-n \cdot k\right)\right. \\
& \left.-\left(q_{1} \cdot k\right)\left(n \cdot p_{1}+n \cdot p_{2}-2 n \cdot k\right)\right],
\end{aligned}
$$

with a related form for $\Delta_{b}^{(12) p e r t}$, found by replacing $q_{1}$ by $-q_{2}$, and hence $s$ by $u$ in the resulting answers. For $\Delta_{a}^{(1)}$, we have

$$
\begin{aligned}
\Delta_{a}^{(1) \text { pert }}= & \frac{1}{2} \Delta_{a}^{(12) \text { pert }} \\
& +8 \int d^{4} k \frac{\delta_{+}\left(k^{2}\right) \delta_{+}\left(\left(p_{1}-k\right)^{2}\right) \delta_{+}\left(\left(p_{2}-k\right)^{2}\right)}{\left(p_{1}-k+q_{1}\right)^{2}}
\end{aligned}
$$




$$
\begin{gathered}
\times\left(e^{(1)} \cdot k\right)(n \cdot k)\left\{\left(e^{(1)} \cdot k\right)\left(n \cdot p_{1}+n \cdot p_{2}-2 k \cdot n\right)\right. \\
\left.+\left(e^{(1)} \cdot n\right)\left(\left(p_{1}-k\right) \cdot\left(p_{2}-k\right)\right)\right\},
\end{gathered}
$$

where we note that the second term is at least quadratic in $k$. The local duality sum rules are found by substituting the results of these

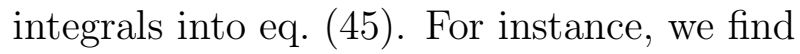

$$
f_{\pi}^{2}\left(H_{1}+H_{2}\right)=\int_{0}^{s_{0}} d s_{1} \int_{0}^{s_{0}} d s_{2} \rho_{4}^{\text {pert }}\left(s_{1}, s_{2}, s, t\right),
$$

where

$$
\rho_{4}^{\text {pert }}\left(s_{i}, s, t\right)=\frac{1}{\left(n \cdot p_{1}\right)\left(n \cdot p_{2}\right)} \Delta_{4}^{(12) p e r t}\left(s_{i}, s, t\right) .
$$

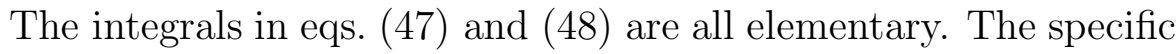
complete integrals and results are given in Appendix C. Here we shall only discuss the leading behavior at fixed angles. It is easy to show that it comes from the term linear in $n \cdot k$, and is specified by

$$
\begin{aligned}
\rho_{4}^{(12) \text { pert }} & \approx \frac{10}{3(2 \pi)^{2}}\left(\frac{s_{1}+s_{2}}{(-t)^{2}}\right)\left(\frac{(s-u)^{2}}{2 s(-u)}\right) \\
& \approx 2 \rho_{4}^{(1) \text { pert }} .
\end{aligned}
$$

Here we have used eq. $\left(\overline{5} 0_{i}^{\prime}\right)$, which defines $\rho$ in terms of $\Delta$, and have combined the contributions from both diagrams of Fig. 5. This result exhibits a comparable power suppression to the spectral weight

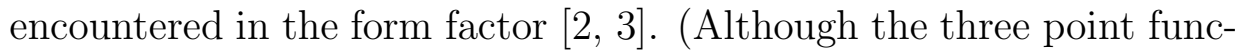
tion involves one less external photon, a factor of $\left(p_{1}+p_{2}\right)^{\lambda}$ is factored from its amplitude, so that the remaining form factor has the same scaling behavior as in Compton scattering.) To be specific, the lowestorder perturbative spectral weight in the latter case may be written as exactly [3제]

$$
\begin{aligned}
\rho_{\pi 3}^{\text {pert }} & \left(s_{1}, s_{2}, t\right) \\
& =\frac{3}{2 \pi^{2}} t^{2}\left(\left(\frac{d}{d t}\right)^{2}+\frac{t}{3}\left(\frac{d}{d t}\right)^{3}\right) \frac{1}{\left(\left(s_{1}+s_{2}-t\right)^{2}-4 s_{1} s_{2}\right)^{1 / 2}} .
\end{aligned}
$$


Asymptotically at large $t$ this function has the characteristic behavior

$$
\rho_{\pi}=\frac{1}{\pi^{2}}\left(\frac{3\left(s_{1}+s_{2}\right)}{t^{2}}+O\left(1 / t^{3}\right)\right)
$$

This is suppressed in $t$ by one additional power compared to the pre-

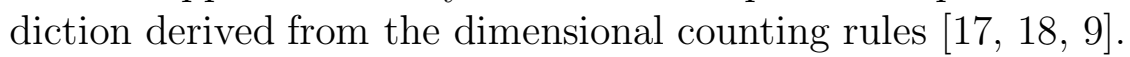

The leading Compton scattering integral in eq. ( $\left(\begin{array}{l}4 \\ 4\end{array}\right)$ can be evaluated trivially in the local duality approximation, in which we neglect $s_{i} / M_{i}^{2}$ compared to unity. We get, for $H_{1}+H_{2}$, for instance,

$$
\begin{aligned}
\left(H_{1}+H_{2}\right) f_{\pi}^{2} & =\frac{10}{3(2 \pi)^{2}} \int_{0}^{s_{0}} d s_{1} \int_{0}^{s_{0}} d s_{2} \frac{\left(s_{1}+s_{2}\right)}{(-t)^{2}}\left(1-\frac{s^{2}+u^{2}}{2 s u}\right) \\
& =\frac{10}{3(2 \pi)^{2}}\left(\frac{s_{0}^{3}}{(-t)^{2}}\right)\left(1-\frac{s^{2}+u^{2}}{2 s u}\right) .
\end{aligned}
$$

As in the case of the form factor, the result is suppressed by an additional power of $t$ compared to the $(1 / t)$ perturbative behavior expected from the dimensional counting rules. This may be understood in terms of the result that the true asymptotic behavior of form factors is dominated by hard gluon exchange [9.9]. It is easy to see (Appendix C) that as $s \rightarrow \infty$, with $s_{1}$ and $s_{2}$ fixed, the momentum of the unscattered parton ( $k$ in Fig. 5 ) is forced to zero, while the incoming and outgoing momenta of the scattered parton approach the momenta of the external "pions". This description of elastic scattering, mediated by a single parton that carries essentially all the energy of a hadron, is often called the "Feynman" mechanism [1] 19.1 . Although it cannot give the true asymptotic behavior, it may be important at intermediate energies $\left[1 \overline{1} \overline{0} \overline{0}_{1}^{\prime}\right]$.

\section{Conclusions}

In this paper, we have shown that QCD sum rules can be formulated for pion Compton scattering, generalizing previous results on meson form factors. We have shown that it is possible to write down a dispersion representation of the scattering amplitude on a finite contour in the complex plane of the external masses, and we have explicitly 
calculated the leading behavior of the spectral function. To derive a sum rule, we found it useful to introduce a modified definition of

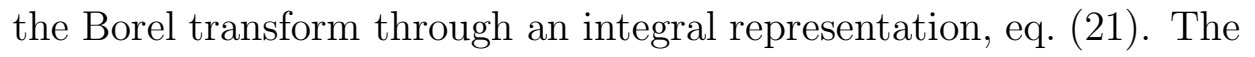
sum rule, eq. (' $\bar{A} \overline{4})$, in the local duality limit, shows suppression by an additional power of $t$ compared to the perturbative result, as shown in eq. ( $\left(\overline{5} \overline{4} \overline{4}_{1}\right)$.

In the future, we hope to pursue the QCD sum rule procedure futher, with the inclusion of power corrections assoicated with the condenstates that appear in power corrections from the operator product expansion. Here, however, we have demonstrated that such a program is possible.

The approach that we have introduced in this paper can also be generalized to the more interesting case of proton Compton scattering $[20]$, where experimental data are availiable, while a direct comparison with perturbative QCD results based on leading power factorization theorems [플ㄹ is possible. Although the leading power result will, by definition, dominate for sufficiently large $t$, we expect the sum rule result to be a valuable tool in the interpretation of realistic experiments. Of particular interest will be the angular dependence of the sum rule result, which may distinguish between leading-power factorization theorems and the "Feynman mechanism" that dominates the sum rule spectral functions. This could help us to understand the roles of these two formalisms for describing hadronic elastic scattering at available energies.

Acknowledgments. This work was supported in part by the National Science Foundation, grants PHY-9211367 and 91080541, by the U.S. Department of Energy under contract DE-AC05-84ER40150, and and by the Texas National Research Laboratory.

\section{A Analyticity and Infrared Safety}

To derive a sum rule for the Compton scattering amplitude, we must be able to prove, first, that the amplitude satisfies the dispersion relation, eq. ( $\left(\overline{1}_{1} \bar{q}_{1}\right)$. In addition, we must check that for nonzero values of

$p_{i}^{2}$ the amplitude is infrared safe; i.e. that its perturbative expansion is free of infrared divergences. Otherwise, the lowest-order term in 
the expansion will be swamped by divergent higher-order corrections. The demonstrations of these two features of the fixed-angle Compton amplitude are related, and we present them together. We will, of course, work in perturbation theory.

Eq. (1) $\left.\bar{q}_{1}^{\prime}\right)$ is consistent with singlularities at positive values of $p_{1}^{2}$ and/or $p_{2}^{2}$, corresponding to normal and anomalous thresholds. Let us recall $\left[\overline{1} \overline{1} \overline{Q_{i}}\right]$ that the condition for a singularity in a Feynman amplitude is that the momentum integrals be "pinched" between coalescing poles from the propagators of internal lines. If internal lines are not on-shell the integrand is finite; if the momentum contours are not pinched, we may deform the momentum integrals into another region where the integrands are finite.

A useful way of characterizing points in momentum space where some set of lines go on-shell is through "reduced diagrams", in which all lines that are off-shell are shrunk to points. Now suppose the resulting reduced diagram represents a point in momentum space at which the contours are truly pinched. Then a theorem due to Coleman and Norton [223i] states that the diagram corresponds to a classical scattering process, in which all vertices correspond to points in spacetime, connected by the motion of on-shell, free, classical particles, whose velocity is determined by their mass and momentum. This theorem turns out to be a very useful tool in the analysis that follows.

Let us start by considering Compton scattering with space-like photons, $q_{i}^{2}<0$. We will relax this condition later. Now suppose $p_{1}^{2}$ is either negative, or complex, but with an amplitude that obeys the restriction,

$$
\left|p_{i}^{2}\right| \leq \lambda^{2}
$$

with $\lambda^{2}=(s+t) / 4$, as in eq. (15), so that

$$
\operatorname{Re}(u)<0 \text {. }
$$

We will be trying to show that for such a $p_{1}^{2}$ the Compton amplitude has cut plane analyticity in the $p_{2}^{2}$ plane when $p_{2}^{2}$ also satisfies eq. ( $\left(\underline{1} \underline{5}_{1}^{\prime} \overline{5}_{1}^{\prime}\right)$.

Let us see what kind of physical-scattering reduced diagrams, which potentially give singularities, can include external currents with momenta in the ranges just described, all the time keeping $s$ and $t$ fixed and large. Because $s$ is positive and $t$ negative, we may assume that 
the energies of $p_{1} \ldots q_{2}$ remain positive. The first vertex in the reduced diagram must therefore result from the scattering of $p_{1}$ and $q_{1}$, or of some subset of particles from one of their virtual states. When $q_{1}^{2}<0$ and $p_{1}^{2}<0$ or complex, however, neither the photon nor $p_{1}$ can decay into only on-shell physical particles as part of the physical process. Since $t<0, p_{1}$ also cannot decay by emitting the (possibly unphysical) momentum $p_{2}$, and because $u<0$ (in the region we are considering), it cannot decay into a physical state by emitting $q_{2}$, even if $p_{1}^{2}$ is real.

We may now take the limit $q_{1}^{2} \rightarrow 0$, which brings us up to, but not beyond, thresholds in the $q_{1}^{2}$ channel. The virtual states into which the incoming photon can "decay" consist of collinear massless particles, which propagate together at the speed of light. In any space-time picture, they therefore act in the same manner as a single particle. The first vertex in the reduced diagram must therefore involve the direct scattering of $q_{1}$ (or its collinear, lightlike decay products) and $p_{1}$ at a point, and all the remaining particles must emerge from that point. Any situation such as this, in which all particles emerge from a single point in space-time, strongly reduces the number of possible reduced diagrams [ $[\overline{2} \overline{4} \bar{i}]$. In fact, in this case, the only possible reduced diagrams corresponding to singular points involve no further "hard" scatterings with nonzero momentum transfer. All further interactions involve the local interactions of massive particles, relatively at rest, of exactly collinear-moving massless particles, and/or the emission and absorption of exactly zero-momentum particles. In our case, this means that we may only have thresholds when $p_{2}^{2}=\left(\sum_{i} m_{i}\right)^{2}$, for some set $m_{i}$ of the masses of virtual particles. Similarly, for $q_{2}^{2} \rightarrow 0$ we may have on-shell massless virtual particles moving exactly parallel to $q_{2}$. We need not consider soft particles that connect lines moving parallel to $p_{2}$ with those moving parallel to $q_{2}$, because, since the photon is color and charge neutral, its couplings to soft particles vanish on-shell [25]. In addition, since we keep $q_{2}^{2}$ fixed, we only need to consider the singularities associated with $p_{2}$, that is, its normal thresholds for $p_{2}^{2} \geq 0$. Clearly, analogous reasoning, working backwards from the final toward the initial state can be used to show that the amplitude has cut-plane analyticity in $p_{1}^{2}$ for $p_{2}^{2}$ fixed and off the real axis. This is the first result we set out to prove. 
Given the preceeding arguments, the proof of infrared safety is not difficult. We are interested in the behavior of the amplitude around the contours at $\left|p_{i}^{2}\right|=\lambda^{2}$. In this region, we want to work in perturbation theory with massless internal lines. It is easy to see that the only reduced diagrams which correspond to points at which the momentum contours are pinched consist of a single hard scattering vertex, and of two "jet" subdiagrams, connected to the external photons, and each consisting entirely of lines collinear to that photon. There may also be zero momentum lines connecting to two jet subdiagrams and/or the hard vertex. Note that because we work to lowest order in QED, the jet subdiagrams are not photon self-energies, but must attach to the hard scattering by at least two finite-momentum lines each. The second result claimed above, the infrared finiteness of such an amplitude, then follows directly from the arguments given in [25] for $e^{+} e^{-}$ annihilation into photons.

\section{B Kinematics}

In this appendix we discuss the kinematics of the pion Compton scattering process in Fig. 3.

Let $q_{1}$ and $q_{2}$ be the momenta of the incoming and outgoing photon, respectively, which are assumed to be on-shell $\left(q_{1}^{2}=q_{2}^{2}=0\right)$. Let also $p_{1}$ and $p_{2}$ be the momenta of the incoming and outgoing pion. The external pion states are off-shell and are characterized by the invariants $s_{1}=p_{1}^{2}$ and $s_{2}=p_{2}^{2}$.

We define as usual the Mandelstam invariants

$$
s=\left(p_{1}+q_{1}\right)^{2}, \quad t=\left(q_{2}-q_{1}\right)^{2}, \quad u=\left(p_{2}-q_{1}\right)^{2},
$$

with the usual relation

$$
s+t+u=s_{1}+s_{2} .
$$

We consider both $s$ and $t$ to be very large and in the physical region $s>0, t<0$.

It is also convenient to introduce light cone variables for the pion and photon momenta as follows:

$$
p_{1}=p_{1}^{+} \bar{v}+p_{1}^{-} v, \quad p_{2}=p_{2}^{+} \bar{v}+p_{2}^{-} v
$$




$$
\begin{gathered}
q_{1}=q_{1}^{+} \bar{v}+q_{1}^{-} v+q_{1 \perp}, \\
\bar{v}=\frac{1}{\sqrt{2}}\left(1,1, \mathbf{0}_{\perp}\right), \quad v=\frac{1}{\sqrt{2}}\left(1,-1, \mathbf{0}_{\perp}\right), \\
q_{1 \perp} \cdot n^{ \pm}=0 .
\end{gathered}
$$

In the definitions of $v$ and $\bar{v}$, the order of components is $(0,3,2,1)$. The momenta of the pions are, in this frame, purely longitudinal. We can choose a particular brick-wall frame in which $p_{1}^{+}=p_{2}^{-}=Q$. Then we have

$$
p_{1}=Q \bar{v}+\frac{s_{1}}{2 Q} v, \quad p_{2}=\frac{s_{2}}{2 Q} \bar{v}+Q v .
$$

A covariant expression for the momentum scale $Q$ can be found from the relation

$$
t=\left(p_{2}-p_{1}\right)^{2}=s_{1}+s_{2}-2 Q^{2}-\frac{s_{1} s_{2}}{2 Q^{2}}
$$

We easily find, by solving for $Q^{2}$,

$$
Q^{2}=\frac{1}{4}\left(s_{1}+s_{2}-t+\delta\right)=\frac{1}{4}(s+u+\delta),
$$

where we have defined

$$
\delta=\sqrt{\left(s_{1}+s_{2}-t\right)^{2}-4 s_{1} s_{2}}=2 Q^{2}-\frac{s_{1} s_{2}}{2 Q^{2}} .
$$

In the scattering process at high energy and fixed angle, $Q^{2}$ is a large parameter. Note that in this notation $t=-2 Q^{2}$ only when $s_{1}=s_{2}=$ 0 . In this frame we also easily get

$$
u=\left(p_{2}-q_{1}\right)^{2}=2 Q^{2}-s+\frac{s_{1} s_{2}}{2 Q^{2}}
$$

Covariant expressions for $q_{1}^{ \pm}$and $q_{2}^{ \pm}$can also be found in the form

$$
\begin{gathered}
q_{1}^{+}=\frac{\left(s-2 Q^{2}\right)\left(2 Q^{2}-s_{2}\right)}{2 Q \delta}, \\
q_{1}^{-}=\frac{\left(2 Q^{2}-s_{1}\right)\left(2 Q^{2} s-s_{1} s_{2}\right)}{4 Q^{3} \delta} .
\end{gathered}
$$


The magnitude of the transverse component $q_{1 \perp}$ of the momentum of the incoming photon is then given by

$$
q_{1 \perp} \equiv q_{\perp}=\frac{\sqrt{(-t)\left(s-2 Q^{2}\right)\left(2 Q^{2} s-s_{1} s_{2}\right)}}{\sqrt{2} Q \delta} .
$$

Similar formulas hold for the momentum of the outgoing photon:

$$
\begin{aligned}
q_{2}^{+} & =\frac{\left(2 Q^{2}-s_{2}\right)\left(2 Q^{2} s-s_{1} s_{2}\right)}{4 Q^{3} \delta} \\
q_{2}^{-} & =\frac{\left(s-2 Q^{2}\right)\left(2 Q^{2}-s_{1}\right)}{2 Q \delta}
\end{aligned}
$$

\section{Computation of the Spectral Weights}

In this appendix, we discuss the evaluation of the integrals in eq. ( $\bar{A} \overline{7} \bar{i})$ and their asymptotic behavior at high energy. The results for the crossed diagram are carried out analogously.

We first note that we may use the identity

$$
2 q_{1} \cdot k=s-s_{1}-\left(p_{1}-k+q_{1}\right)^{2}
$$

to rewrite eq. $\left(\bar{M}_{\overline{4}} \overline{\bar{T}}_{1}\right)$ in terms of integrals for the fully on-shell box and triangle diagrams that are no more than quadratic in $k^{\mu}$ in the numerator. Thus, we have

$$
\begin{aligned}
\Delta_{a}^{(12) p e r t}= & 16\left(q_{1} \cdot p_{1}+q_{1} \cdot p_{2}\right) \int d^{4} k I_{s}^{(4 a)}\left((n \cdot k)\left(n \cdot p_{1}\right)-(n \cdot k)^{2}\right) \\
& +8 \int d^{4} k\left[I_{s}^{(3)}-\left(s-s_{1}\right) I_{s}^{(4 a)}\right] \\
& \times\left((n \cdot k)\left(n \cdot p_{1}+n \cdot p_{2}\right)-2(n \cdot k)^{2}\right)
\end{aligned}
$$

where $I^{(3)}$ and $I_{s}^{(4 a)}$ are the integrands for the double discontinuities of the triangle and box diagrams, respectively,

$$
\begin{aligned}
I_{s}^{(3)} & =\delta_{+}\left(k^{2}\right) \delta_{+}\left(\left(p_{1}-k\right)^{2}\right) \delta_{+}\left(\left(p_{2}-k\right)^{2}\right) \\
I_{s}^{(4 a)} & =\frac{\delta_{+}\left(k^{2}\right) \delta_{+}\left(\left(p_{1}-k\right)^{2}\right) \delta_{+}\left(\left(p_{2}-k\right)^{2}\right)}{\left(p_{1}-k+q_{1}\right)^{2}} .
\end{aligned}
$$


The relevant integrals are now

$$
\begin{aligned}
v^{\mu} & =\int I_{s}^{(3)} k^{\mu} \\
V^{\mu \nu} & =\int I_{s}^{(3)} k^{\mu} k^{\nu}
\end{aligned}
$$

for the triangle and

$$
\begin{aligned}
w_{a}^{\mu} & =\int I_{s}^{(4 a)} k^{\mu} \\
W_{a}^{\mu \nu} & =\int I_{s}^{(4 a)} k^{\mu} k^{\nu}
\end{aligned}
$$

for the box.

Before discussing the foregoing integrals, it is useful to describe the corresponding scalar integrals for the scalar triangle and the scalar box. For the triangle we have

$$
\Delta_{3}=\int d^{4} k \delta_{+}\left(k^{2}\right) \delta_{+}\left(\left(p_{1}-k\right)^{2}\right) \delta_{+}\left(\left(p_{2}-k\right)^{2}\right)=\frac{\pi}{2 \delta},
$$

where $\delta$ has been defined in eq. $\left(i_{1} \overline{6} \overline{5}\right)$. The integral for $\Delta_{3}$ can be simply evaluated by going to the brick-wall frame defined above; $2 \delta$ appears as the Jacobian of the transformation from the components $k^{\mu}$ to the variables $k^{2}, p_{1} \cdot k$, and $p_{2} \cdot k$, leaving one trivial angular integral,

$$
\Delta_{3}=\frac{1}{4 \delta} \int_{0}^{2 \pi} d \phi
$$

with $\phi$ the angle in transverse space. Interestingly, the discontinuity on the cut is given by the same result for massive as for massless quarks.

For the scalar box diagrams in Figs. 5(a) and 5(b), three internal lines are on shell, while the upper internal line is off-shell,

$$
\Delta_{a}^{s c}\left(s_{1}, s_{2}, s, t\right)=\int d^{4} k \frac{\delta_{+}\left(k^{2}\right) \delta_{+}\left(\left(p_{1}-k\right)^{2}\right) \delta_{+}\left(\left(p_{2}-k\right)^{2}\right)}{\left(p_{1}-k+q_{1}\right)^{2}} .
$$

Three angular integrals are trivially done using the change of variables mentioned above, but the remaining angular integral is now nontrivial,

$$
\Delta_{a}^{s c}\left(s_{1}, s_{2}, s, t\right)=\frac{1}{4 \delta} \int_{0}^{2 \pi} d \phi\left(\frac{1}{s-s_{1}-2\left(q_{1 L} \cdot k_{L}-q_{\perp} k_{\perp} \cos \phi\right)}\right),
$$


where we define

$$
q_{1 L} \cdot k_{L} \equiv q_{1}^{+} k^{-}+q_{1}^{-} k^{+},
$$

which is a number that is fixed in the brick-wall frame according to the values given in Appendix B and the mass shell delta functions (see below). The angular integral is a standard one,

$$
\int_{0}^{2 \pi} d \phi\left(\frac{1}{A+B \cos \phi}\right)=\frac{2 \pi}{\left(A^{2}-B^{2}\right)^{1 / 2}} .
$$

For the particular constants

$$
\begin{aligned}
& A=s-s_{1}-2 q_{1 L} \cdot k_{L}=\frac{(-t)}{\delta^{2}}\left[s(s+u)-2 s_{1} s_{2}\right], \\
& B=2 q_{\perp} k_{\perp}=\frac{2(-t)}{\delta^{2}}\left[s(-u)+s_{1} s_{2}\right]^{1 / 2}\left[s_{1} s_{2}\right]^{1 / 2},
\end{aligned}
$$

we find the following, rather unobvious, identity,

$$
\frac{1}{\left(A^{2}-B^{2}\right)^{1 / 2}}=\frac{1}{\left(\left[s-s_{1}-2 q_{1 L} \cdot k_{L}\right]^{2}-\left[2 q_{\perp} k_{\perp}\right]^{2}\right)^{1 / 2}}=\frac{\delta}{s(-t)} .
$$

Thus, at lowest order, the box and crossed box are given by the remarkably simple forms,

$$
\begin{aligned}
& \Delta_{a}=-\frac{\pi}{2 s t}, \\
& \Delta_{b}=-\frac{\pi}{2 u t},
\end{aligned}
$$

where we have used the crossing relation between the two.

In deriving the spectral weight for the box diagram with fermions, it is useful to know expressions for the components of $k^{\mu}$ in the brickwall frame kinematics of Appendix B,

$$
\begin{aligned}
& k^{+}=\frac{s_{2}\left(Q-s_{1} / 2 Q\right)}{\delta}, \\
& k^{-}=\frac{s_{1}\left(Q-s_{2} / 2 Q\right)}{\delta},
\end{aligned}
$$

as fixed by the delta functions in the integral. We notice that both components are small and $O(1 / Q)$. Similarly, we have the simple expression

$$
k_{\perp}^{2}=\frac{s_{1} s_{2}(-t)}{\delta^{2}} .
$$


Note that all the components of $k^{\mu}$ vanish in the limit $Q \rightarrow \infty$, as is appropriate for the Feynman mechanism (Section 5).

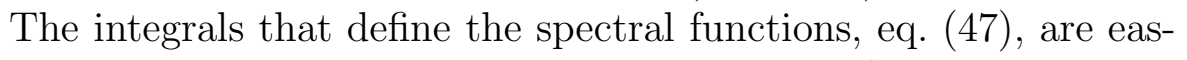
ily reduced in the brick-wall frame to the factor $1 / 4 \delta$ times angular integrals which generalize eq. ( $\left(\overline{8} \overline{8} \overline{1}_{1}^{1}\right)$,

$$
I_{a}=\int_{0}^{2 \pi} d \phi\left(\frac{\cos ^{a} \phi}{A+B \cos \phi}\right)
$$

with $a=0,1,2$ and with $A$ and $B$ given as above. For completeness, we list the elementary results,

$$
\begin{aligned}
& I_{0}=\frac{2 \pi}{\left(A^{2}-B^{2}\right)^{1 / 2}}=\frac{2 \pi \delta}{s(-t)}, \\
& I_{1}=\frac{2 \pi A}{B\left(A^{2}-B^{2}\right)^{1 / 2}}-\frac{2 \pi}{B}=\frac{4 \pi \delta}{s^{2}(-t)} \frac{\sqrt{s(-u) s_{1} s_{2}+s_{1}^{2} s_{2}^{2}}}{4 Q^{2}-2 s_{1} s_{2} / s} \\
& I_{2}=\frac{2 \pi A^{2}}{B^{2}\left(A^{2}-B^{2}\right)^{1 / 2}}-\frac{2 \pi A}{B^{2}}=\frac{2 \pi \delta}{s^{2}(-t)} \frac{s(s+u)-2 s_{1} s_{2}}{4 Q^{2}-2 s_{1} s_{2} / s} .
\end{aligned}
$$

We note that in the frame we work, $A \gg B$, and that while $I_{0}$ and $I_{2}$ behave as $1 / A$ for large $A, I_{1}$ goes as $1 / A^{2}$. In terms of these expressions, we find,

$$
\begin{gathered}
v^{\mu}=\frac{\pi}{\delta^{3}}\left[s_{2}\left(p_{1} \cdot p_{2}-s_{1}\right) p_{1}^{\mu}+s_{1}\left(p_{1} \cdot p_{2}-s_{2}\right) p_{2}^{\mu}\right] \\
V^{\mu \nu}=\frac{2 \delta}{\pi}\left(v^{\mu} v^{\nu}-\frac{1}{2} d^{\mu \nu} v^{2}\right)
\end{gathered}
$$

where $d^{11}=d^{22}=1$, with all other components zero, and

$$
\begin{gathered}
w^{\mu}=\frac{\delta}{s(-t)} v^{\mu}+\frac{1}{2 \delta} \sqrt{\frac{s_{1} s_{2}}{s(-u)+s_{1} s_{2}}} q_{\perp}^{\mu}, \\
W^{\mu \nu}=\frac{2 s(-t)}{\pi}\left(w^{\mu} w^{\nu}-\delta_{\mu 2} \delta_{\nu 2}\left(w_{2}\right)^{2}\right) \\
-\frac{s_{1} s_{2}(-t)}{4 \delta^{3}}\left(I_{2} \delta_{\mu 2} \delta_{\nu 2}+\left(I_{0}-I_{2}\right) \delta_{\mu 1} \delta_{\nu 1}\right) .
\end{gathered}
$$




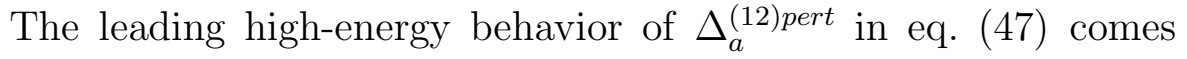
entirely from the term

$$
\begin{aligned}
\Delta_{a}^{(12) p e r t} & =8(s-u)\left(n \cdot w_{a}\right)\left(n \cdot p_{1}\right)+\cdots \\
& \sim 4(s-u)\left(\frac{\pi}{s(-t)^{2}}\left(s_{1}+s_{2}\right)\right)\left(n \cdot p_{1}\right)\left(n \cdot p_{2}\right),
\end{aligned}
$$

where we have used $\left(n \cdot p_{1}\right)=\left(n \cdot p_{2}\right)$. Note that by eq. ( $\left(\overline{5}_{0}^{-1}\right)$, the factor $\left(n \cdot p_{1}\right)\left(n \cdot p_{2}\right)$ cancels in the sum rule. Similarly, we find for the crossed diagram $\Delta_{b}^{(12) \text { pert }}$ :

$$
\Delta_{b}^{(12) p e r t} \sim 4(u-s)\left(\frac{\pi}{u(-t)^{2}}\left(s_{1}+s_{2}\right)\right)\left(n \cdot p_{1}\right)\left(n \cdot p_{2}\right) .
$$

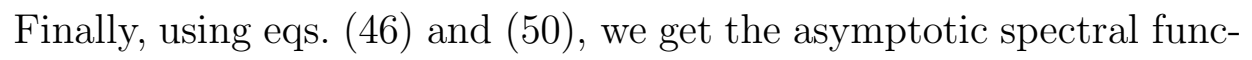
tion quoted in eq. ( $(\underline{\underline{5}} \overline{1} \overline{1})$. 


\section{References}

[1] M.A. Shifman, A. I. Vainshtein and V.I. Zakharov, Nucl. Phys. B147 (1979) $385,448,519$.

[2] B.L. Ioffe and A.V. Smilga, Phys. Lett. 114B (1982) 353; Nucl. Phys. B216 (1983) 373.

[3] V.A. Nesterenko and A.V. Radyushkin, Phys. Lett. 115B (1982) 410; JETP Lett. 35 (1982) 488.

[4] V.M. Belyaev and B.L. Ioffe, Nucl. Phys. B310 (1988) 548.

[5] L.L. Enkovskii and B. V. Struminskii, Theo. and Math. Phys. 57 (1983) 979 (Teor. Mat. Fiz. 57 (1983) 41).

[6] A.A. Logunov, L. D. Soloviev and A. N. Tavkelidze, Phys. Lett. B24 (1967) 181.

[7] J.J. Sakurai, Phys. Lett. 46B (1973) 207.

[8] De Alfaro, S. Fubini, G. Furlan and C. Rossetti, Currents in Hadron Physics (North-Holland, 1973).

[9] G.P. Lepage and S.J. Brodsky, Phys. Lett. 87B (1979) 359; Phys. Rev. Lett. 43 (1979) 545; A.V. Efremov and A.V. Radyushkin, Phys. Lett. 94B (1980) 245; S.J. Brodsky and G.P. Lepage, in Perturabtive Quantum Chromodynamics, ed. A.H. Mueller (World Scientific, Singapore, 1989).

[10] A.V. Radyushkin, Acta Phys. Polon. B15 (1984) 403.

[11] N. Isgur and C.H. Llewellyn Smith, Phys. Rev. Lett. 52 (1984) 1080; Nucl. Phys. B317 (1989), 526.

[12] H.-N. Li and G. Sterman, Nucl. Phys. B381 (1992) 129.

[13] R. Akhoury, Phys. Rev. D19 (1979) 1250.

[14] G.R. Farrar, G. Sterman and H. Zhang, Phys. Rev. Lett. 62 (1989) 2229.

[15] V.B. Berestetskii, E.M. Lifshitz and L.P. Pitaevskii, Relativistic Quantum Theory (Pergamon Press, 1971), p. 235. 
[16] R.J. Eden, P.V. Landshoff, D.I. Olive and J.C. Polkinghorne, The Analytic S-matrix (Cambridge Univ. Press., 1966).

[17] V.A. Matveev, R.M. Muradyan and A.N. Tavkhelidze, Lett. Nuovo Cimento 7 (1973) 719.

[18] S.J. Brodsky and G.R. Farrar, Phys. Rev. Lett. 31 (1973) 1153.

[19] R.P. Feynman, Photon-Hadron Interactions, (W.A. Benjamin, Reading, MA, 1972), p. 145.

[20] C. Corianò, in preparation.

[21] E. Maina and G.R. Farrar, Phys. Lett. 206B (1988) 120; G. R. Farrar and H. Zhang, Phys. Rev. D41 (1990) 3348; 42 (1990) 2413(E).

[22] A.S. Kronfeld and B. Nižić, Phys. Rev. D44 (1991) 3445.

[23] S. Coleman and R.E. Norton, Nuovo Cimento Ser. 10, 38 (1965) 438.

[24] G. Sterman, Phys. Rev. D17 (1978) 2773.

[25] G. Sterman, Phys. Lett. 73B (1978) 440. 


\section{Figure Captions}

1. The integration contours for the Borel transform in the $p_{i}^{2}$ plane, eq. (ini

2. Lowest order perturbative contribution to the pion form factor.

3. The pion Compton scattering amplitude.

4. The integration contours for the Compton scattering amplitude. Here $\gamma$ is the dispersion contour of eq. (' $\left(\underline{6}_{-}^{i}\right)$, and $\Gamma$ the transform contour of eq. ( $(2 \sqrt[1]{1} \overline{1})$.

5. Lowest order contributions to the spectral weight for Compton scattering. 